\title{
Editorial
}

Digestion

Published online: August 17, 2005

DOI: $10.1159 / 000087601$

\section{Probiotics: A New Treatment for Antibiotic-Associated Diarrhea?}

\author{
Rémy F. Meier \\ Department of Gastroenterology, Hepatology and Clinical Nutrition, University Hospital, Kantonsspital Liestal, \\ Liestal, Switzerland
}

Antibiotic-associated diarrhea is a frequent side effect of broad-spectrum antibiotics. The clinical presentation of diarrhea reaches from mild to fulminant pseudomembranous Clostridium difficile toxin-induced colitis. The frequency of antibiotic-associated diarrhea can affect as many as $25 \%$ of patients. In the last decade, there has been an increase of antibiotic-associated diarrhea of 500\%. In up to $80 \%$, the cause of diarrhea is not clear [1]

One of the mechanisms for the development of antibiotic-associated side effects seems to be related to an alteration in the intestinal flora. The intestinal flora protects the individual against enteral pathogens by multiple mechanisms: (a) production of bacteriocins; (b) competitive inhibition of binding to the mucosa; (c) enhancing synthesis of mucus, and (d) secretion of secretory $\operatorname{IgA}$ [2].

Probiotics (life-viable, non-pathogen microbial organisms) have been shown to have immune modulatory properties and to enhance the mucosal barrier. In fact, probiotics have anti-bacterial, anti-viral and anti-inflammatory properties and restore the intestinal flora balance; they were first used for the treatment or prevention of antibiotic-associated diarrhea. Several probiotics, such as Lactobacillus acidophilus, Lactobacillus caseii, Lactobacillus rhamnosus GG and the yeast Saccharomyces boulardii have been evaluated. The clear benefits of probiotics are still debated and the data are conflicting, since many of the trials were small and with an open-label design. D'Souza et al. [3] performed a meta-analysis includ- ing 9 randomized, double-blind, placebo-controlled trials studying the efficacy of probiotics in this setting. In these trials, 3 different probiotics (S. boulardii, Lactobacillus spp. and Enterococcus faecium) were used. The odds ratio for diarrhea to occur was similar for all species. The combined odds ratio was 0.37 (95\% confidence interval 0.26 $0.53 ; \mathrm{p}<0.01)$ in favor of active treatment over placebo. This analysis has been criticized because of its inconsistent definition of diarrhea and that more than half of the studies did not analyze the data on an intention-to-treat basis. The second meta-analysis by Cremonini et al. [4] included only 7 studies with more consistent definitions of outcome. The results were similar. The combined relative risk was 0.39 (95\% confidence interval 0.27-0.57). According to these two meta-analyses the results infer that the use of Lactobacillus spp. and S. boulardii in the prevention of antibiotic-associated diarrhea are beneficial.

In this issue of Digestion Hawrelak et al. [5] report in a new systematic review of the effect of a single strain of L. rhamnosus GG in preventing the onset of antibioticassociated diarrhea. This report reflects the inherent problems of this topic. Although 25 clinical trials have been published so far, only 6 placebo-controlled trials could be included. 18 trials included probiotics other than L. rhamnosus GG and one trial was not placebo-controlled. Of the 6 trials, 4 used different antibiotics in children $(n=2)$ and adults $(n=2)$. These studies were also different in sample size, duration and doses of the co-

\section{KARGER \\ Fax +41613061234 E-Mail karger@karger.ch} www.karger.com
(C) 2005 S. Karger AG, Basel 0012-2823/05/0721-0049\$22.00/0

Accessible online at: www.karger.com/dig
Rémy F. Meier, MD

Department of Gastroenterology, Hepatology and Clinical Nutrition

University Hospital, Kantonsspital Liestal

CH-4410 Liestal (Switzerland)

Tel. +41 6192521 87, Fax +41 6192528 04, E-Mail remy.meier@ksli.ch 
treatment with probiotics. There were only 2 homogeneous studies looking at Helicobacter pylori eradication with small numbers of patients included; the same dose and duration of probiotics and the same antibiotics were administered. The reported trials in this systematic review were published in the period ranging from 1990 to 2002 with different methodological qualities (Jadad score between 1 and 5 ). With these limits in mind, the authors could confirm the findings of the previously reported meta-analysis. In 4 of 6 trials a significant reduction in the risk of antibiotic-associated diarrhea was noted with the co-administration of L. rhamnosus GG. Unfortunately, the study with the largest sample size undertaken in hospitalized patients was negative. One potential explanation for these results could be that these patients were sicker than in the other studies, which included only ambulatory patients.

In the evolutionary battle against infectious gastrointestinal disease, probiotics seem to be useful agents to prevent or treat these diseases. Very interesting is the prevention of antibiotic-associated diarrhea. Several other publications (not included in the previous meta-analysis or in the systematic review of Hawrelak et al. [5]) have recently been published. In a double-blind, placebo-controlled study involving 150 elderly patients with an antibiotic regimen, a probiotic mixture of Lactobacillus and
Bifidobacterium showed a significant reduction in C. difficile toxin in the probiotic group compared to the placebo group (46 vs. 78\%) [5]. With respect to diarrhea, the incidence of samples positive for C. difficile-associated toxin was $2.9 \%$ in the probiotic group compared to $7.25 \%$ in the placebo group [6]. Erdeve et al. [7] tested the effect of probiotics in children receiving an antibiotic treatment. 462 children aged $1-5$ years receiving sulbactamampicillin or azithromycin were randomized to the treatment with $S$. boulardii or antibiotics alone. Antibiotic-associated diarrhea was found in $19 \%$ receiving an antibiotic treatment alone and in $6 \%$ with the additional probiotic treatment. Finally, a recently published study performed with Bacillus clausii reduced the incidence of nausea, diarrhea and epigastric pain associated with a triple anti-H. pylori therapy as compared to placebo [8].

One major limitation of all published results is the inhomogeneous and variable definition of diarrhea. In the future, to overcome these problems, it is absolutely mandatory to perform trials with adequate power using the same probiotics with the same doses and duration using a standard and uniform definition of diarrhea. Such trials should demonstrate that a co-treatment with probiotics will definitely reduce antibiotic-associated side effects and reduce health care costs.

\section{References}

1 Hurley BW, Nguyen CC: The spectrum of pseudomembranous enterocolitis and antibiotic-associated diarrhea. Arch Intern Med 2002;162:2177-2184

2 Elmer G W, McFarland LV: Biotherapeutic agents in the treatment of infectious diarrhea. Gastroenterol Clin North Am 2001;30:837854

3 D'Souza AL, Rajkumar C, Cooke J, Bulpitt CJ: Probiotics in prevention of antibiotic associated diarrhoea: meta-analysis. BMJ 2002;324: 1361-1366.
4 Cremonini F, Di Caro S, Nista EC, Bartolozzi F, Capelli G, Gasbarrini G, Gasbarrini A: Meta-analysis: the effect of probiotic administration on antibiotic-associated diarrhoea. Aliment Pharmacol Ther 2002;16:1461-1467.

5 Hawrelak JA, Whitten DL, Myers SP: Is Lactobacillus rhamnosus GG effective in preventing the onset of antibiotic-associated diarrhea: A systematic review. Digestion 2005;72:5156.

6 Plummer S, Weaver MA, Harris JC, Dee P, Hunter J: Clostridium difficile pilot study: effects of probiotic supplementation on the incidence of $C$. difficile diarrhoea. Int Microbiol 2004;7:59-62.
7 Erdeve O, Tiras U, Dallar Y: The probiotic effect of Saccharomyces boulardii in a pediatric age group. J Trop Pediatr 2004;50:234-236.

8 Nista E C, Candelli M, Cremonini F, Cazzato I A, Zocco M A, Franceschi F, Cammarota G, Gasbarrini G, Gasbarrini A: Bacillus clausii therapy to reduce side-effects of anti-Helicobacter pylori treatment: randomized, doubleblind, placebo controlled trial. Aliment Pharmacol Ther 2004;20:1181-1188. 